\title{
HYPERCYCLIC WEIGHTED SHIFTS
}

\author{
HÉCTOR N. SALAS
}

Dedicated to Domingo A. Herrero, in memoriam

\begin{abstract}
An operator $T$ acting on a Hilbert space is hypercyclic if, for some vector $x$ in the space, the orbit $\left\{T^{n} x: n \geq 0\right\}$ is dense. In this paper we characterize hypercyclic weighted shifts in terms of their weight sequences and identify the direct sums of hypercyclic weighted shifts which are also hypercyclic. As a consequence, we show within the class of weighted shifts that multi-hypercyclic shifts and direct sums of fixed hypercyclic shifts are both hypercyclic. For general hypercyclic operators the corresponding questions were posed by $D$. A. Herrero, and they still remain open. Using a different technique we prove that $I+T$ is hypercyclic whenever $T$ is a unilateral backward weighted shift, thus answering in more generality a question recently posed by K. C. Chan and J. H. Shapiro.
\end{abstract}

\section{INTRODUCTION}

A bounded operator $T$ on a Hilbert space $H$ is cyclic if there exists an $x \in H$ such that its orbits under the operator

$$
\operatorname{orb}(T, x)=\left\{x, T x, T^{2} x, T^{3} x, \ldots\right\},
$$

generates a dense linear manifold. If $\operatorname{orb}(T, x)$ is itself dense in $H$, then $T$ is hypercyclic. Such an $x$ is called a hypercyclic vector for $T$. The literature on cyclic operators is very extensive. In contrast, the literature on hypercyclic operators is relatively scarce.

In [14], Rolewicz was the first to isolate the concept of hypercyclicity. $\mathrm{He}$ actually considered continuous linear operators on an $F$-space, i.e., a complete metrizable topological linear space.

In [5], Gethner and Shapiro also studied hypercyclicity on $F$-spaces. Their main result, [5, Theorem 2.2], gives a useful criterion for an operator $T$ to be hypercyclic. As a consequence of this result, they were able to obtain simultaneously two theorems on entire functions; namely, G. D. Birkhoff's translation theorem and G. R. Mac Lane's differentiation theorem. (See also the generalization of this result by Godefroy and Shapiro [6, Theorem 5.1].) They also proved in [5] that very general Bergman backward shifts are hypercyclic.

Received by the editors October 18,1993; orginally communicated to the Proceedings of the $A M S$ by Palle E. T. Jorgensen.

1991 Mathematics Subject Classification. Primary 47B37, 47B99.

Key words and phrases. Cyclic and hypercyclic vectors, bilateral weighted shifts, unilateral backward weighted shifts. 
Shields proved that whenever $T$ is cyclic then its set of cyclic vectors is a $G_{\delta}$, [16, Proposition 40]. It was later shown by Herrero that when the set of cyclic vectors of $T$ is a closed subset of $H \backslash\{0\}$, then it is either $H \backslash\{0\}$ or nowhere dense in $H$ [8, Proposition 2.1(iii)]. A natural example of such behavior is $M_{z}$, multiplication by $z$, on $A^{2}(U)$, the Bergman space of holomorphic functions of the unit disc $U$. Cyclic functions for $M_{z}$ vanish nowhere on $U$, and they are not dense in $A^{2}(U)$. (For more information see [6, Remark 3.2].) What happens when $T$ is hypercyclic? Several people have shown, in different contexts, that the set of its hypercyclic vectors is a $G_{\delta}$ which is dense in $H$. (See, for instance, [4], [5] and [13].)

An even more striking difference between the class of cyclic operators and the subclass of hypercyclic ones is the following fact. Finite-dimensional spaces support many cyclic operators; but in [13, Theorem 1.2], Kitai showed that finite-dimensional spaces cannot support hypercyclic operators.

From now on we will work on infinite-dimensional separable Hilbert spaces. The symbol $\oplus$ means orthogonal direct sum. If $T=T_{1} \oplus T_{2}$ is hypercyclic, then it is easy to show that each summand is hypercyclic. However, $T$ need not be hypercyclic if $T_{1}$ and $T_{2}$ are. (Actually $T$ need not be even cyclic [10], [15].)

Herrero's Problem 1 in [11] is: Does $T$ hypercyclic imply that $T \oplus T$ is also hypercyclic?

Conjecture 1 of [11] says: If there exists $\left\{x_{1}, \ldots, x_{n}\right\}$ such that the $\bigcup_{k=1}^{n} \operatorname{orb}\left(T, x_{k}\right)$ is dense in $H$, then $T$ is hypercyclic. In other words, the conjecture is that multi-hypercyclic operators are hypercyclic.

Assume now that $H=l^{2}(Z)$. The operator $T$ is a bilateral (forward) weighted shift with respect to the canonical basis $\left\{e_{n}: n \in Z\right\}$ if $T e_{n}=a_{n} e_{n+1}$, where the weight sequence $\left\{a_{n}: n \in Z\right\}$ is a bounded subset of $C \backslash\{0\}$. (Without loss of generality we may, and will, assume that each $a_{n}$ is positive.) The class of weighted shifts is a favorite testing ground for operator-theorists. (See for example the classical survey by Shields [16].)

In section 2 we characterize the hypercyclic bilateral weighted shifts in terms of their weight sequences. Several consequences are obtained from this result; among them we show that the answer to both Problem 1 and Conjecture 1 of [11] is affirmative for the class of bilateral weighted shifts and the class of unilateral backward weighted shifts. In section 3 we prove, using a different technique, that $I+T$ is hypercyclic whenever $T$ is a unilateral backward weighted shift. This section was motivated by a question of Chan and Shapiro in [3, page 1447].

\section{WeIGHTED SHIFTS}

The first result is basic for all that follows in this section. The conditions on the weights of a hypercyclic bilateral weighted shift $T$ can be obtained by inspecting the action of the powers of $T$ on the span $\left\{e_{k}: a \leq k \leq b\right\}$ (where $\left\{e_{k}: k \in Z\right\}$ is the canonical basis of $l^{2}(Z)$ ). On the other hand, if the conditions on the weights are satisfied, then hypercyclic vectors can be assembled in a piecemeal fashion.

Theorem 2.1. Let $T$ be a bilateral weighted shift with positive weight sequence $\left\{a_{n}\right\}$. Then $T$ is hypercyclic if and only if given $\varepsilon>0$ and $q \in N$, there exists 
$n$ arbitrarily large such that for all $|j| \leq q$

$$
\prod_{s=0}^{n-1} a_{s+j}<\varepsilon \text { and } \prod_{s=1}^{n} a_{j-s}>1 / \varepsilon .
$$

Proof. Assume that $T$ is hypercyclic. Since the set of hypercyclic vectors for $T$ is dense, there is a hypercyclic vector $x$ for $T$ such that

$$
\left\|x-\sum_{|j| \leq q} e_{j}\right\|<\delta \text {. }
$$

We can also find $n$ arbitrarily large, $n>2 q$, such that

$$
\left\|T^{n} x-\sum_{|j| \leq q} e_{j}\right\|<\delta
$$

In terms of the coefficients of $x$ inequality (1) implies that $\left|\left\langle x, e_{j}\right\rangle\right|>1-\delta$ if $|j| \leq q$ and $\left|\left\langle x, e_{j}\right\rangle\right|<\delta$ otherwise. Now assume that $\delta<1$. Since $n>2 q$, inequality (2) implies that for $|j| \leq q$

$$
\left\|T^{n}\left(\left\langle x, e_{j}\right\rangle e_{j}\right)\right\|=\left(\prod_{s=0}^{n-1} a_{j+s}\right)\left|\left\langle x, e_{j}\right\rangle\right|<\delta .
$$

Thus

$$
\prod_{s=0}^{n-1} a_{j+s}<\delta /\left|\left\langle x, e_{j}\right\rangle\right|<\delta /(1-\delta) .
$$

On the other hand, inequality (2) implies that $\left\|T^{n}\left\langle x, e_{j-n}\right\rangle e_{j-n}-e_{j}\right\|<\delta$ for $|j| \leq q$. This in turn implies $\left|\prod_{s=1}^{n} a_{j-s}\left\langle x, e_{j-n}\right\rangle-1\right|<\delta$. Therefore

$$
\prod_{s=1}^{n} a_{j-s}>(1-\delta) /\left|\left\langle x, e_{j-n}\right\rangle\right|>(1-\delta) / \delta .
$$

By choosing $\delta$ such that $\delta /(1-\delta)<\varepsilon$, we see that the conditions on the weights are necessary if $T$ is hypercyclic.

To see the converse we will prove a lemma which is interesting in its own right. Its method of proof is similar to the one used in [15].

Lemma 2.2. Let $T$ be a bilateral weighted shift. Assume that if $\varepsilon>0$ and vectors $g, h$ are in the span of $\left\{e_{j}:|j| \leq q\right\}$, then there would exist an $n$ arbitrarly large and $a$ vector $u$ in the span of $\left\{e_{j}:-q-n \leq j \leq q-n\right\}$ such that

$$
\begin{gathered}
\|u\|<\varepsilon, \\
\left\|T^{n}(u)-g\right\|<\varepsilon, \\
\left\|T^{n}(h)\right\|<\varepsilon .
\end{gathered}
$$

Then $T$ is hypercyclic.

Proof. We will explicitly exhibit a hypercyclic vector $f$ for $T$. (Also observe that conditions (i) and (ii) imply that $\|T\|>1$.) 
Assume that $\left\{g_{k}=\sum_{|j| \leq k}\left\langle g_{k}, e_{j}\right\rangle e_{j}: k \in N\right\}$ is dense in $l^{2}(Z)$. We will construct $f$ to be equal to $\sum_{k=1}^{\infty} f_{k}$, where

$$
\lim _{k \rightarrow \infty}\left\|T^{n_{k}}\left(f_{k}\right)-g_{k}\right\|=0
$$

and $n_{k}$ is a rapidly increasing sequence to be specified.

Let $n_{1}=0$ and $f_{1}=g_{1}$. Assume that for $1 \leq j \leq k$, the number $n_{j}$ and the vector $f_{j}$ in the span of $\left\{e_{i}:-j-n_{j} \leq i \leq j-n_{j}\right\}$ have been chosen. We now choose $n$ and $u$ by applying the hypothesis to $\varepsilon=M^{-n_{k}} 2^{-k-1}$ and the vectors $g=g_{k+1}, h=f_{1}+\cdots+f_{k}$, where $M=\|T\|$.

Let the $n$ and $u$ so obtained be denoted by $n_{k+1}$ and $f_{k+1}$ respectively. We also ask that $n_{k}+\sum_{i=1}^{k+1} i<n_{k+1}$ to ensure that the supports of the $f_{i}$ 's are pairwise disjoint. Then

$$
\begin{gathered}
\left\|f_{k+1}\right\|<M^{-n_{k}} 2^{-k-1}, \\
\left\|T^{n_{k+1}}\left(f_{k+1}\right)-g_{k+1}\right\|<M^{-n_{k}} 2^{-k-1},
\end{gathered}
$$

and

$$
\left\|T^{n_{k+1}}\left(\sum_{j=1}^{k} f_{j}\right)\right\|<M^{-n_{k}} 2^{-k-1} .
$$

We are now ready to verify that $f=\sum f_{j}$ is hypercyclic. It follows that

$$
\begin{aligned}
\left\|T^{n_{k}}\left(\sum_{j=1}^{\infty} f_{j}\right)-g_{k}\right\| & \leq\left\|T^{n_{k}}\left(\sum_{j=1}^{k-1} f_{j}\right)\right\|+\left\|T^{n_{k}}\left(f_{k}\right)-g_{k}\right\|+\sum_{j=k+1}^{\infty}\left\|T^{n_{k}}\left(f_{j}\right)\right\| \\
& \leq M^{-n_{k-1}} 2^{-k}+M^{-n_{k-1}} 2^{-k}+\sum_{j=k+1}^{\infty} M^{n_{k}}\left\|f_{j}\right\| \\
& \leq 2^{-k+2} \text {. }
\end{aligned}
$$

We can now show the remaining implication in Theorem 2.1. First we observe that if $f=\sum_{|j| \leq q}\left\langle x, x_{j}\right\rangle e_{j}$, then

$$
\left\|T^{n} f\right\| \leq \max \left\{\prod_{k=0}^{n-1} a_{j+k}:|j| \leq q\right\}\|f\| .
$$

But such a vector $f$ is also in the domain of the (possibly unbounded) operator $T^{-n}$, and it satisfies

$$
\left\|T^{-n} f\right\| \leq \max \left\{\left(\prod_{k=1}^{n} a_{j-k}\right)^{-1}:|j| \leq q\right\}\|f\| .
$$

Let $\varepsilon>0$ and $q \in N$. Assume there is $n>2 q$ which satisfies $\prod_{s=0}^{n-1} a_{j+s}<\varepsilon$ and $\prod_{s=1}^{n} a_{j-s}>1 / \varepsilon$ for all $|j| \leq q$. If vectors $g, h$ are in the span $\left\{e_{j}:|j| \leq\right.$ $q\}$, then inequalities (3) and (4) imply that

$$
\left\|T^{-n} g\right\| \leq \varepsilon\|g\| \text { and }\left\|T^{n} h\right\| \leq \varepsilon\|h\| .
$$

By setting $T^{-n} g=u$, we see that the three conditions in the hypothesis of Lemma 2.2 are satisfied. Thus $T$ is hypercyclic and the proof of Theorem 2.1 is completed. 
Remark. Hypercyclic bilateral backward weighted shifts can be characterized in a similar fashion; they are unitarily equivalent to forward shifts. In Theorem 2.8 we will see unilateral backward weighted shifts as compressions of bilateral backward weighted shifts.

Corollary 2.3. There exists an hypercyclic operator $T$ such that $T^{*}$ is also hypercyclic.

Proof. Since $T^{*}$ is a weighted shift if $T$ is so, it suffices to construct $\left\{a_{z}: z \in\right.$ $Z\}$ such that $\left\{a_{z}\right\}$ and $\left\{b_{z}=a_{-z}\right\}$ both satisfy the conditions of Theorem 2.1 .

The above result was obtained in [15]. The shift used as an example in [15] has an almost symmetrical weight sequence, i.e., $a_{n}=a_{-n}$, except for the rapidly increasing sequence $\left\{n_{k}\right\}$. However, no hypercyclic shifts with symmetrical weights can exist.

Corollary 2.4. If $T$ is a weighted shift such that its weight sequence $\left\{a_{n}\right\}$ satisfies $a_{n}=a_{-n}$, then $T$ is not hypercyclic.

Proof. Since $a_{n}=a_{-n}$ the inequalities $a_{0} a_{1} \cdots a_{n-1}<\varepsilon$ and $a_{n} a_{n-1} \cdots a_{1}>$ $1 / \varepsilon$ imply that $a_{n}>a_{0} /(\varepsilon)^{2}$. Thus $\varepsilon^{2}>a_{0} /\|T\|$ cannot be arbitrarily small.

The arguments for proving Theorem 2.1 can be easily adapted to the case of a direct sum of a finite number of (not necessarily equal) bilateral weighted shifts.

Theorem 2.5. Let $T_{i}$ be bilateral weighted shifts with $T_{i} e_{n}=a_{n, i} e_{n+1}$. Then $\bigoplus_{i=1}^{m} T_{i}$ is hypercyclic if and only if given $\varepsilon>0$ and $q \in N$ there exists $n$ large enough such that for all $|j| \leq q$

$$
\max \left\{\prod_{k=0}^{n-1} a_{j+k, i}: 1 \leq i \leq m\right\}<\varepsilon \text { and } \min \left\{\prod_{k=1}^{n} a_{j-k, i}: 1 \leq i \leq m\right\}>1 / \varepsilon .
$$

The following result is also known. (See [11] and [15].)

Corollary 2.6. There exist hypercyclic operators $S, T$ such that $S \oplus T$ is not hypercyclic.

Proof. Choose sequences $\left\{a_{z}: z \in Z\right\}$ and $\left\{b_{z}: z \in Z\right\}$, each one satisfying the condition in Theorem 2.1, but not satisfying the condition together by peaking out of phase. In particular, we can construct $\left\{a_{z}\right\},\left\{b_{z}\right\}$ such that $\max \left\{\prod_{k=0}^{s} a_{k}, \prod_{k=0}^{s} b_{k}\right\}>1$ for any natural number $s$. Then set $S$ and $T$ to be the corresponding weighted shifts. An application of Theorem 2.5 yields the result.

We would like to give the unilateral version of Theorem 2.5. A unilateral backward weighted shift $T$ is defined by $T e_{n}=a_{n} e_{n-1}$ if $n>0$ and by $T e_{0}=0$, where $\left\{e_{n}: n \in Z^{+}\right\}$is the canonical basis of $l^{2}\left(Z^{+}\right)$.

Remark. A unilateral forward shift can never be hypercyclic. Indeed, let $T$ acting on $l^{2}\left(Z^{+}\right)$be defined by $T e_{n}=a_{n} e_{n+1}$. Let $x$ be an arbitrary vector; the orthogonal projection of $\operatorname{orb}(T, x)$ on the span of $\left\{e_{k}: k<n\right\}$ consists of at most $n$ vectors. 
Proposition 2.7. Let $T$ be a hypercyclic operator on $H$. Assume that $B$ is an invariant subspace of $T^{*}$. Then the compression of $T$ to $B$ is hypercyclic on $B$.

Proof. Recall that the compression of $T$ to $B$ is defined by PTP restricted to $B$, where $P$ is the orthogonal projection on $B$. Since $B$ is invariant under $T^{*}$, the subspace $B^{\perp}$ is invariant under $T$; in other words $P T(I-P)=0$. Therefore

$$
T^{n}=(P T P)^{n}+\sum_{k=1}^{n}((I-P) T)^{k}(P T)^{n-k} P+((I-P) T(I-P))^{n} .
$$

The last equality implies that for any pair of vectors $x \in H$ and $b \in B$

$$
\left\|(P T P)^{n} x-b\right\| \leq\left\|T^{n} x-b\right\| .
$$

Thus if $u$ is a hypercyclic vector for $T$, $\operatorname{orb}(T, P u)$ is dense in $B$.

Theorem 2.8. For $1 \leq i \leq m$, let $T_{i}$ be unilateral backward weighted shifts with positive weight sequences $\left\{a_{n, i}: n \in Z^{+}\right\}$. Then $\bigoplus_{i=1}^{m} T_{i}$ is hypercyclic if and only if $\sup \left\{\min \left\{\prod_{s=1}^{n} a_{s, i}, 1 \leq i \leq m\right\}: n \in N\right\}=\infty$.

Proof. We prove it for the case of just one unilateral backward shift. Take $T$ as the compression to $l^{2}\left(Z^{+}\right)$of a bilateral backward weighted shift whose weights corresponding to nonpositive indexes are 1/2. An application of Theorem 2.1 and Proposition 2.7 yields the result.

We will now apply this result to the case of $m=1$. Let $\beta=\{\beta(k): k \geq 0\}$ be a sequence of positive numbers for which

$$
\sup \{\beta(k) / \beta(k+1): k \geq 0\}<\infty .
$$

In [16], $H^{2}(\beta)$ denotes the space of formal series $f(z)=\sum_{k=0}^{\infty} \hat{f}(k) z^{k}$ for which

$$
\|f\|_{\beta}^{2}=\sum_{k=0}^{\infty}|\hat{f}(k)|^{2} \beta(k)<\infty .
$$

The backward shift $B$ defined on $H^{2}(\beta)$ by $B f(z)=\sum_{k=0}^{\infty} \hat{f}(k+1) z^{k}$ is bounded since the weights are $(\beta(k) / \beta(k+1))^{1 / 2}$. We now have

Corollary 2.9. Let $B$ be a backward weighted shift as above. Then $B$ is hypercyclic if and only if $\lim \inf \beta(k)=0$.

This result was obtained by Gethner and Shapiro when $\{\beta(k)\}$ is decreasing [5, Theorem 4.1]. In this case the backward shifts can be seen as very general Bergman backward shifts; in particular, the sequence $\beta(k)=1 /(k+1)$ corresponds to the Bergman backward shift.

In what follows, "shift" stands for either "bilateral shift" or "unilateral backward shift". We can now partially answer Herrero's questions mentioned in the introduction.

Corollary 2.10. Let $T$ be a hypercyclic weighted shift. Then $T \oplus T \oplus \cdots \oplus T$ is also hypercyclic.

Proof. For the bilateral case apply Theorem 2.5 and for the unilateral backward case apply Theorem 2.8 . 
Corollary 2.11. If $T$ is a multi-hypercyclic weighted shift, then $T$ is hypercyclic. Proof. We will only prove the case of a bilateral shift. Let $x_{1}, \ldots, x_{k}$ be such that $\bigcup_{i=1}^{k} \operatorname{orb}\left(T, x_{i}\right)$ is dense in $l^{2}(Z)$. Then there exists a vector, say $x_{1}$, and sequences $n_{s}$ and $q_{s}$ such that $\left\|T^{n_{s}} x_{1}-\sum_{|j|<q_{s}} e_{j}\right\|<1 / 2^{s}$. By arguing as we did in the first part of Theorem 2.1, we see that the weights of $T$ satisfy the conditions for hypercyclicity.

In [13], Kitai asks whether $T$ hypercyclic implies that $T^{n}$ is also hypercyclic. Our next observation gives a partial affirmative answer to Kitai's question.

Corollary 2.12. Let $T$ be a weighted shift. If $T$ is hypercyclic so is any power of $T$.

Proof. $T^{n}$ is a direct sum of $n$ weighted shifts that satisfies the requirements of either Theorem 2.5 or Theorem 2.8.

\section{IDENTITY PLUS A UNILATERAL BACKWARD WEIGHTED SHIFT IS HYPERCYCLIC}

In this section we will show that $I+T$ is hypercyclic whenever $T$ is a unilateral backward shift with positive weights. This result goes beyond the inquiry by Chan and Shapiro [3], in which they ask whether $I+D$ is hypercyclic. In their context, $D$ is the derivative operator acting on spaces of "slowly growing" entire functions. Proposition 1.1 of [3] shows that $D$ is a unilateral backward weighted shift.

The following lemma might be known since it is about a Toeplitz matrix, but we have been unable to locate a reference. We provide its proof for the sake of completeness.

Lemma 3.1. Let $A=\left(a_{i j}\right)$ be the $2^{k} \times 2^{k}$ matrix with $a_{i j}=\frac{1}{\left(2^{k}+j-i\right) !}$. Then $A$ is invertible.

Proof. We will show that $\operatorname{det} A \neq 0$. If we multiply the $i$ th row by $\left(2^{k+1}-i\right)$ ! we get the matrix $B$ :

$$
B=\left(\begin{array}{ccccc}
\prod_{l=1}^{2^{k}-1}\left(2^{k}+l\right) & \prod_{l=2}^{2^{k}-1}\left(2^{k}+l\right) & \cdots & \left(2^{k+1}-1\right) & 1 \\
\prod_{l=0}^{2^{k}-2}\left(2^{k}+l\right) & \prod_{l=1}^{2^{k}-2} 2^{k}+l & \cdots & \left(2^{k+1}-2\right) & 1 \\
\vdots & \vdots & \ddots & \vdots & \vdots \\
\prod_{l=3}^{2^{k}+1} l & \prod_{l=4}^{2^{k}+1} l & \cdots & \left(2^{k}+1\right) & 1 \\
\prod_{l=2}^{2^{k}} l & \prod_{l=3}^{2^{k}} l & \cdots & 2^{k} & 1
\end{array}\right) .
$$

Assertion. If the powers of 2 are factored out in each column of $B$, then the main diagonal consists of odd numbers. Moreover, in each column the entries below the main diagonal are even.

Let $C=\left(c_{i j}\right)$ be the resulting matrix, and assume the above assertion is true. Since $C$ has been obtained from $A$ by performing elementary row and column operations, $\operatorname{det} A$ is nonzero if and only if $\operatorname{det} C$ is so. But

$$
\operatorname{det} C=\sum_{\sigma}(-1)^{\operatorname{sgn} \sigma} \prod_{i=1}^{2^{k}} c_{i \sigma(i)}
$$

and every summand is even except $\prod_{i=1}^{2^{k}} c_{i i}$ which is odd. Thus $\operatorname{det} C$ is odd. 
We will now prove the assertion. The number $\frac{\prod_{l=1}^{n}(m+l)}{n !}$ is the combinatory number $\left(\begin{array}{c}m+n \\ n\end{array}\right)$ and therefore an integer. In particular this means that we can factor out at least as many factors of 2 from $\prod_{l=1}^{n}(m+l)$ as from $n$ !. For $1 \leq j \leq 2^{k}-1$, we have that $b_{j j}=\prod_{l=1}^{2^{k}-j}\left(2^{k}+l\right)$, and observe that we can factor out the same number of factors of 2 from $2^{k}+l$ that from $l$. Thus we can factor out the same number of factors 2 from $b_{j j}$ that from $\left(2^{k}-j\right)$ !.

Assume that $i<j$. Since $b_{i j}=\prod_{l=j-i+1}^{2^{k}-i}\left(2^{k}+l\right)$, we can factor out the same number of factors 2 from $b_{i j}$ that from $\prod_{l=j-i+1}^{2^{k}-i} l$. It follows from this and the observation about the combinatory numbers that we can factor out at least as many factors 2 from $b_{i j}$ as from $b_{j j}$.

Now let $i>j$ and compare $b_{i j}$ to $b_{j j}$. (So $b_{i j}$ is below the main diagonal entry.) After simplifying it suffices to compare $\prod_{l=1-i+j}^{0}\left(2^{k}+l\right)$ to $\prod_{l=2^{k}-i+1}^{2^{k}-j}\left(2^{k}+l\right)$. But again it suffices to look only at $(i-j-1) ! 2^{k}$ and $\prod_{l=2^{k}-i+1}^{2^{k}-j} l$. Let $2^{s}$ be the largest power of 2 between $2^{k}-i+1$ and $2^{k}-j$. Then $\prod_{l=2^{k}-i+1}^{2^{k}-j} l$ has the same number of factors of 2 as $2^{s}\left(2^{k}-2^{s}-j\right) !\left(i+2^{s}-2^{k}-1\right) !$.

To finish the proof of the assertion, observe that $2^{k-s}\left(\begin{array}{c}i-j-1 \\ 2^{k}-2^{s}-j\end{array}\right)$ is even.

Remark. Peter Miletta pointed out the following: If the matrix $B$ in Lemma 3.1 is rotated by $\pi / 2$, its determinant is $W\left(t^{2^{k+1}-1}, \ldots, t^{2^{k}}\right)$, the Wronskian of the linearly independent functions $t^{k^{k+1}-1}, \ldots, t^{2^{k}}$, evaluated at $t=1$. In order to show that $\operatorname{det} B \neq 0$, it is enough to find a linear differential equation of order $2^{k}$ for which such functions are solutions and 1 is a regular point. The canonical way of building such a differential equation is to set $W\left(y, t^{2^{k+1}-1}, \ldots, t^{2^{k}}\right)=0$. The resulting differential equation is of an Euler-Cauchy type, and it has order $2^{k}$ only if $\operatorname{det} B \neq 0$, which is precisely what has to be proved. Consequently, if this method of proving that $\operatorname{det} B \neq 0$ is to be followed, another differential equation would be needed.

We still need a technical lemma in preparation for the theorem in this section.

Lemma 3.2. Let $C_{n}=\left(c_{i j}(n)\right)$ be the $2^{k} \times 2^{k}$ matrix whose entries $c_{i j}(n)$ are the combinatory numbers $\left(\begin{array}{c}n \\ 2^{k}+j-i\end{array}\right)$. Let $B_{n}=\left(b_{i}(n)\right)$ be a column vector such that $b_{i}(n)$ is a polynomial in $n$ of degree at most $2^{k}-i$, where $i=1, \ldots, 2^{k}$.

Then for $n$ large enough there is a solution $X_{n}=\left(x_{i}(n)\right)$ of the equation $B_{n}=C_{n} X_{n}$ and the entries $x_{i}(n)$ satisfy $\left|x_{i}(n)\right| \leq P / n^{i}$, where $P$ is a constant. Proof. Since

$$
\operatorname{det} C_{n}=\sum_{\sigma}(-1)^{\operatorname{sgn} \sigma} \prod_{i=1}^{2^{k}}\left(\begin{array}{c}
n \\
2^{k}+\sigma(i)-i
\end{array}\right)
$$

each summand is a polynomial in $n$ of degree

$$
\sum_{i=1}^{2^{k}}\left(2^{k}+\sigma(i)-i\right)=2^{2 k}+\left(\sum_{i=1}^{2^{k}} \sigma(i)-i\right)=2^{2 k}
$$

Thus $\operatorname{det} C_{n}$ is also a polynomial of degree $2^{2 k}$ with leading coefficient equal to $\operatorname{det} A$, where $A$ is the matrix of Proposition 3.1. Thus if $n$ is large enough 
$\operatorname{det} C_{n} \neq 0$ and so $C_{n}$ is invertible. Let $C_{n}^{i}$ be the matrix obtained from $C_{n}$ after replacing its $i$ th column by $B_{n}$. Since

$$
\operatorname{det} C_{n}^{i}=\sum_{\sigma}(-1)^{\operatorname{sgn} \sigma} \prod_{t \neq s, \sigma(t) \neq i}\left(\begin{array}{c}
n \\
2^{k}+\sigma(t)-t
\end{array}\right) b_{s}(n)
$$

each summand is a polynomial in $n$ of degree at most

$$
2^{k}-s+\sum_{t \neq s}\left(2^{k}+\sigma(t)-t\right)=2^{2 k}-i
$$

thus $\operatorname{det} C_{n}^{i}$ is a polynomial of degree at most $2^{2 k}-i$.

By Cramer's rule $x_{i}(n)=\operatorname{det} C_{n}^{i} / \operatorname{det} C_{n}$, and therefore $\left|x_{i}(n)\right| \leq P / n^{i}$.

We now have all the necessary tools to prove the main result of this section, but first some comments are in order. Since $T$ is a unilateral backward weighted shift, the spectrum of $I+T$ is a disc centered at 1 , thus satisfying Kitai's criterion that the spectrum of a hypercyclic operator must meet the unit circle. (This includes the case when the spectrum is just the singleton $\{1\}$. ) On the other hand, the work of Chan-Shapiro makes clear that $I+D$ is hypercyclic when its spectrum is a nontrivial disc. (See for instance "Large Hilbert Spaces" on page 1447 of [3]. Recall that $D$ is the derivative operator.) Thus, their question mentioned at the beginning of this section boils down to whether $I+D$ is hypercyclic when $D$ is quasi-nilpotent. It should also be noted that the work of Godefroy-Shapiro suggests that hypercyclicity is easier for functions of backward shifts when there is a rich supply of eigenvalues. (See sections 4 and 5 of [6].) This is exactly what happens in Chan-Shapiro's setting: If the spectrum of $I+D$ is a nontrivial disc, then its interior contains a nontrivial disc of eigenvalues. For an arbitrary unilateral backward shift $T$ the situation is more complicated: $T$ may be non-quasi-nilpotent and still have zero as its only-and simple-eigenvalue [16, Theorem 8].

The proof of our result does not involve any heavy machinery, and it treats all unilateral backward weighted shifts on an equal footing. The elementary nature of the proof is somewhat surprising since the analogous result of Chan and Shapiro about the hypercyclicity of translations [3, Theorem 2.1] uses a fine manipulation of function theory and the Gethner-Kitai-Shapiro criterion [5, Theorem 2.2].

Theorem 3.3. Let $T$ be defined by $T e_{i}=w_{i} e_{i-1}$ if $i \geq 1$ and $T e_{0}=0$, where the weights are positive. Then $I+T$ is hypercyclic.

Proof. Let $\left\{z_{k}=\sum_{i=0}^{2^{k}-1} z_{i, k} e_{i}: k \in N\right\}$ be dense in $l^{2}\left(Z^{+}\right)$. Let us begin the proof by first considering this problem: Find numbers $x_{1}, x_{2}$ and $n$ such that

$$
\left\|(I+T)^{n}\left(x_{1} e_{2}+x_{2} e_{3}\right)-z_{1}\right\| \leq 1 / 2 \text {. }
$$

Recall that

$$
T^{m} e_{s}= \begin{cases}\prod_{l=s-m+1}^{s} w_{l} e_{s-m}, & \text { if } m \leq s \\ 0, & \text { otherwise }\end{cases}
$$

This implies that

$$
\begin{aligned}
(I+T)^{n}\left(x_{1} e_{2}+x_{2} e_{3}\right)= & \left(x_{1}+\left(\begin{array}{c}
n \\
1
\end{array}\right) w_{3} x_{2}\right) e_{2}+x_{2} e_{3}+\left(\left(\begin{array}{c}
n \\
1
\end{array}\right) T+\left(\begin{array}{c}
n \\
2
\end{array}\right) T^{2}\right) x_{1} e_{2} \\
& +\left(\left(\begin{array}{c}
n \\
2
\end{array}\right) T^{2}+\left(\begin{array}{c}
n \\
3
\end{array}\right) T^{3}\right) x_{2} e_{3} .
\end{aligned}
$$


Therefore

$$
\begin{aligned}
&\left\|(I+T)^{n}\left(x_{1} e_{2}+x_{2} e_{3}\right)-z_{1}\right\|^{2} \\
&=\left\|\left(x_{1}+\left(\begin{array}{c}
n \\
1
\end{array}\right) w_{3} x_{2}\right) e_{2}+x_{2} e_{3}\right\|^{2} \\
&+\left\|\left(\left(\begin{array}{c}
n \\
1
\end{array}\right) T+\left(\begin{array}{c}
n \\
2
\end{array}\right) T^{2}\right) x_{1} e_{2}+\left(\left(\begin{array}{c}
n \\
2
\end{array}\right) T^{2}+\left(\begin{array}{c}
n \\
3
\end{array}\right) T^{3}\right) x_{2} e_{3}-z_{1}\right\|^{2} .
\end{aligned}
$$

Making the second summand on the right-hand side of the above equality be zero is equivalent to

$$
w_{2} w_{1}\left(\begin{array}{l}
n \\
2
\end{array}\right) x_{1}+w_{3} w_{2} w_{1}\left(\begin{array}{l}
n \\
3
\end{array}\right) x_{2}=z_{0,1}
$$

and

$$
w_{2}\left(\begin{array}{c}
n \\
1
\end{array}\right) x_{1}+w_{3} w_{2}\left(\begin{array}{c}
n \\
2
\end{array}\right) x_{2}=z_{1,1}
$$

Since

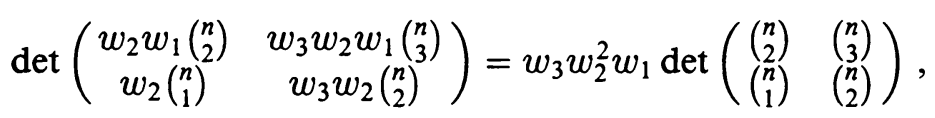

Lemma 3.2 says that we can choose an $n$ large enough such that $\left|x_{1}\right| \leq P / n$ and $\left|x_{2}\right| \leq P / n^{2}$ where $P$ is a constant. Therefore inequality (1) is satisfied. We set such an $n=n_{1}$ and relabel $x_{1}, x_{2}$ as $a_{2}, a_{3}$ respectively. Thus, we have $n_{1}$ and $y_{1}=a_{2} e_{2}+a_{3} e_{3}$.

We will find a rapidly increasing sequence $n_{j}$ and vectors $y_{j}=\sum_{i=2^{j}}^{2^{j+1}-1} a_{i} e_{i}$ such that

$$
\left\|y_{j}\right\|<2^{-j-1}(I+\|T\|)^{-n_{j}-1}
$$

and

$$
\left\|(I+T)^{n_{j}}\left(\sum_{s=1}^{j} y_{s}\right)-z_{j}\right\| \leq 2^{-j} .
$$

A calculation similar to the one made in Lemma 2.2 then shows that the vector $y=\sum_{j=1}^{\infty} y_{j}$ is hypercyclic for $I+T$.

Assume that we have already chosen $n_{1}<\cdots<n_{k-1}$ and $y_{1}, \ldots, y_{k-1}$ satisfying (2) and (3). Let $D=\left(d_{i j}\right)$ be the $2^{k} \times 2^{k}$ matrix with entries

$$
d_{i j}=\left(\begin{array}{c}
n \\
2^{k}+j-i
\end{array}\right) \prod_{s=i}^{2^{k}+j-1} w_{s} \text {. }
$$

Then

$$
\operatorname{det} D=\left(\prod_{j=-2^{k}+1}^{2^{k}-1}\left(w_{2^{k}+j}\right)^{2^{k}-|j|}\right) \operatorname{det} C
$$

where $C=\left(c_{i j}\right)$ and $c_{i j}=\left(\begin{array}{c}n \\ 2^{k}+j-i\end{array}\right)$. By applying Lemma 3.2, it follows that if $n$ is large enough the system

$$
\left\langle(I+T)^{n}\left(\sum_{j=1}^{k-1} y_{j}+\sum_{i=1}^{2^{k}} x_{i} e_{2^{k}+i-1}\right)-z_{k}, e_{p}\right\rangle=0,
$$


with $p=0, \ldots, 2^{k}-1$, satisfies the condition $\left|x_{i}\right| \leq P / n^{i}$, for some constant $P$. Since

$$
(I+T)^{n}\left(\sum_{j=1}^{k-1} y_{j}+\sum_{i=1}^{2^{k}} x_{i} e_{2^{k}+i-1}\right)-z_{k}=\sum_{p=1}^{2^{k}}\left(\sum_{j=0}^{p-1}\left(\begin{array}{l}
n \\
j
\end{array}\right) T^{j}\right) x_{p} e_{2^{k}+p-1},
$$

it follows that

$$
\left\|(I+T)^{n}\left(\sum_{j=1}^{k-1} y_{j}+\sum_{i=1}^{2^{k}} x_{i} e_{2^{k}+i-1}\right)-z_{k}\right\| \leq \sum_{p=1}^{2^{k}}\left(\sum_{j=0}^{p-1}\left(\begin{array}{c}
n \\
j
\end{array}\right)\|T\|^{j}\right)\left\|x_{p}\right\| \leq L / n
$$

where $L$ is a new constant.

So we can choose an $n=n_{k}$ that satisfies (2) and (3) and relabel $x_{i}$ as $a_{2^{k}+i-1}$. We have then obtained $y_{k}$, and therefore the proof of the theorem is now completed.

\section{CONCLUDING REMARKS}

Many of the results obtained in this paper can be extended to weighted shifts on $l^{p}(Z), l^{p}\left(Z^{+}\right)$where $1 \leq p<\infty$; the proofs remain essentially the same.

There are still many intriguing problems on hypercyclicity, among which are the ones posed by Rolewicz. For instance, Problem 1 in [14]: Does each infinite-dimensional separable Banach space support a hypercyclic operator? In [10], Herrero characterized the closure of the hypercyclic operators acting on a Hilbert space in terms of spectral properties. So what happens for Banach spaces? Halperin, Kitai and Rosenthal showed in [7] that any linearly independent denumerable set of vectors in a separable Hilbert space is contained in the orbit of a hypercyclic operator. They asked whether this result remains true for separable Banach spaces; an affirmative answer to this would also provide an affirmative answer to Rolewicz's problem 1.

\section{ACKNOWLEDGMENT}

The material in this paper was to be included in a joint paper with Domingo A. Herrero. The untimely death of Domingo cut short this project. I will always remember Domingo as a teacher, colleague, and above all as a great friend.

\section{REFERENCES}

1. C. Apostol, L. A. Fialkow, D. A. Herrero, and D. Voiculescu, Approximation of Hilbert space operators, Vol. II, Research Notes in Math., vol. 102, Pitman, Boston, London, and Melbourne, 1984.

2. P. S. Bourdon and J. H. Shapiro, Cyclic composition operators in $H^{2}$, Proc. Sympos. Pure Math., vol. 51, Part 2, Amer. Math. Soc., Providence, RI, 1990.

3. K. C. Chan and J. H. Shapiro, The cyclic behavior of translation operators on Hilbert spaces of entire functions, Indiana Univ. Math. J. 40 (1991), 1421-1449.

4. S. M. Duyos-Ruis, Universal functions of the structure of the space of entire functions, Soviet Math. Dokl. 30 (1984), no. 3, 713-716.

5. R. M. Gethner and J. H. Shapiro, Universal vectors for operators on spaces of holomorphic functions, Proc. Amer. Math. Soc. 100 (1987), 281-288. 
6. G. Godefroy and J. H. Shapiro, Operators with dense, invariant, cyclic vector manifolds, J. Funct. Anal. 98 (1991), 229-269.

7. I. Halperin, C. Kitai, and P. Rosenthal, On orbits of linear operators, J. London Math. Soc. 31 (1985), 561-565.

8. D. A. Herrero, Possible structures for the set of cyclic vectors, Indiana Univ. Math. J. 28 (1979), 913-926.

9. —_, Approximation of Hilbert space operators, Vol. I, 2nd ed., Pitman Research Notes in Math. Ser., vol. 224, Longman Sci. Tech., Harlow and Wiley, New York, 1989.

10. __ Limits of hypercyclic and supercyclic operators, J. Funct. Anal. 99 (1991), 179-190.

11. __ Hypercyclic operators and chaos, J. Operator Theory 28 (1992), 93-103.

12. D. A. Herrero and Z. Y. Wang, Compact perturbation of hypercyclic and cyclic operators, Indiana Univ. Math. J. 3 (1990), 819-829.

13. C. Kitai, Invariant closed sets for linear operators, Thesis, Univ. of Toronto, 1982.

14. S. Rolewicz, On orbits of elements, Studia Math. 32 (1969), 17-22.

15. H. Salas, $A$ hypercyclic operator whose adjoint is also hypercyclic, Proc. Amer. Math. Soc. 112 (1991), 765-770.

16. A. L. Shields, Weighted shifts operators and analytic function theory, Topics of Operator Theory, Math. Surveys Monographs, vol. 13, Amer. Math. Soc., Providence, RI, 1974, pp. $49-128$.

Departamento de Matemáticas, Universidad de Puerto Rico, Mayagüez, 00681-5000 PUERTO RICO

E-mail address: h_salas@rumac.upr.clu.edu 